\title{
Experimental investigations and control of mechanical systems dynamics
}

\author{
Jan Awrejcewicz $^{1}$ - Pawel Olejnik ${ }^{1}$
}

Published online: 6 May 2015

(c) The Author(s) 2015. This article is published with open access at Springerlink.com

\section{Erratum}

The thematic issue of International Journal of Dynamics and Control published in March 2015 was edited by the guest editors, Professors Jan Awrejcewicz and Paweł Olejnik of the Lodz University of Technology in Lodz, Poland. The title of the thematic issue is "Experimental investigations and control of mechanical systems dynamics". We have inadvertently mislabeled the title of the thematic issue published in March 2015. Moreover, the preface attached below written by the guest editors Professors Jan Awrejcewicz and Paweł Olejnik is for the thematic issue published in March 2015.

\section{Preface to the thematic issue published in March 2015}

Dynamic properties of miscellaneous mechanical systems have a direct influence on the form of their dynamical responses, which are affected by non-compensated inaccuracies of the structural parameters, noisy disturbances, or an instability of the systems. A precise dynamical analysis of mechatronic systems extended on real mechanisms, machines, multibody elastic connections, and even biomechanical systems plays crucial role in the development of optimized solutions. Bearing this in mind, there are extended in this thematic issue selected papers presented during the international conference on "Dynamical systems-Theory and Applications" hold in December 2-5, 2013 in Lodz, Poland. Main areas of modern experimental as well as numerical analysis covered by the issue could be enumerated:

\footnotetext{
Jan Awrejcewicz

jan.awrejcewicz@p.lodz.pl

1 The Lodz University of Technology, Lodz, Poland
}

bifurcations and chaos in dynamical systems, stability of dynamical systems, original numerical methods of vibration analysis, non-smooth systems, engineering systems and differential equations, control in dynamical systems, asymptotic methods in nonlinear dynamics, vibrations of lumped and continuous systems, dynamics in life sciences and bioengineering. A brief description of contents of this thematic issue follows.

Passive energy absorbers used today like complex of bumpers or passenger lifts buffers typically allow to the safe dissipation of energy within a certain range of loads. In the case of high-impact loading variability is desirable to use an adaptive energy absorption system capable of rapid change their dynamic characteristics. The main issue in the analysis of interactions impact of dynamic loads on objects is dissipation of kinetic energy during impact. With regard to that, Zach proposed a description of a model of internal damping. For example, elastomer material EPUNIT is used to build a model of a hyper-deformable material.

Micro-turbines of power output ranging between 1 and $20 \mathrm{~kW}$ are applied in modern dissipated power generation systems. The rotating system presented by Łagodziński et al. is supported in oil-free, airfoil bearings. Compliant surface foil gas bearings are a class of hydrodynamic bearings that use the ambient gas as their working fluid and thus require no dedicated lubrication systems, which makes their design much simpler. Authors present the development and experimental verification of a theoretical numerical model of the foil bearing for analysis of its dynamic characteristics. There has been confirmed between others, that the nature of elastically supported bush motion can dramatically affect dynamic characteristics of the bearing. Therefore, nonlinear modeling of dynamic properties, including design characteristics of the support and the generated friction forces, becomes indispensable in high-speed foil bearing applications. 
Physical and mathematical model of a 3D double physical pendulum being coupled by two universal joints is studied by Ludwicki et al. The work consists of mathematical modelling, computer simulation, and partially, the experimental analysis to confirm the numerical simulations. The experimental setup realizes kinetic excitation by a non-constant periodic torque of servomotor controlled by the computer. Presented exemplary results showed a number of non-linear effects, including chaos, quasi-periodic and periodic dynamics.

Experimental measurements focused on the investigation of a fibre behavior are performed by Polach et al. on an assembled weigh-fibre-pulley-drive mechanical system. The time histories of the weight position and the force acting in the fibre are subject to measurement. The same system is numerically investigated by means of a multibody model. The influence of the model parameters on the coincidence of results of experimental measurements and the simulations results are evaluated. The simulations aim is to create a phenomenological model of a fibre, which will be utilizable in fibre modelling in the case of more complicated mechanical or mechatronic systems.

Böhm et al. investigate a vibration driven planar locomotion of mobile robots. The dynamic behavior of a simple tensegrity structure is nonlinear, due to large vibration amplitudes and friction between robot and environment, and is mainly influenced by the magnitude of preliminary stress. The nonlinear equations of motion are derived and transient dynamic analyses are performed, including the consideration of chaotic system behavior near to the primary and secondary eigen-frequencies. The movement behavior depends on the actuation parameters and on the preliminary stress, which are discussed with a focus on single-actuated systems with minimal control effort.

The influence of a selection of AC drives parameters at start-up and braking phases of the transport system with the roller conveyors is discussed by Hetmańczyk and Świder. A wide range of possibilities connected with forming of dynamic characteristics of AC motors reveals a large number of industrial applications taking advantage of an inverter control. Authors have proved, that the decisive factor in the damage prevention of drive components is the correct choice of dynamic parameters, monitoring, rapid diagnosis and removal of failure causes.

Fritzkowski and Walczak apply a MEBDFV solver to the dynamic simulation of some specific multibody systems. Performance of the MEBDFV solver is examined in terms of the algorithmic energy conservation. The test is based on an appropriately formulated relation between the kinetic energy and work. A simplified mathematical model of the human head-cervical spine system served as the model. In a numerical experiment the model is used to analyze motion of the head-neck during rear-end impact which may lead to a whiplash injury.

A problem of a bounded space with the vibroacoustical source inside a generating acoustic field is investigated by Błażejewski. The two aspects of the work relate either to the semi-analytical modelling of an acoustic field or to the minimization of significant factors number required to control the level of an acoustic pressure inside a bounded space. It is clearly seen that an acoustic field inside could be controlled by proper impedance distribution. This knowledge is useful in the passive control of an acoustic pressure level in the enclosure, in the acoustical design in the optimization process, and especially in topology optimization. It allows one to reduce the number of design variables and simplify the problem in some cases.

Constitutive equations form a material model. However, such equations are the addition, which constructs a mathematically solvable system of equations. With regard to that, Béda has focused on the stability problems and the effects of discretization on material modeling, as well studying of the linear mapping operator of the discrete dynamical system. He presents how the discretization, stability and anticipation act on one another. An incursive nature of a material model that leads to instability has been shown.

Żółtowski and Kostek concerned on the inspection of bifurcation diagrams and parameter identification of linear and nonlinear basic systems of machinery. Most of the state measures acquired in the presented experiments required a reduction of the measurement with the use of the procedure of statistic evaluation of separate measures or SVD taken into consideration in multidimensional approaches. Optimized set of symptoms is a basis for a causal effect, most often regressive for multidimensional models.

A lumped mass mechanical model of a thorax subject to a blast pressure wave is taken into consideration by Olejnik and Awrejcewicz. A thorax spring-dashpot model developed by Lobdell is implemented in numerical modeling of dynamics of the multibody system. The five degrees of freedom mechanical model of a chest adjacent to the elastic backrest is subject to an impulse loading generated by the blast pressure wave released by an explosion. The so-called coupling of the pressure wave to the thorax is reconsidered. With respect to the evident existence of inherent time delays of displacements, the system of coupled bodies is described by a time delay differential equations that are derived from the large-scale systems approach. Numerical solutions present interesting dynamical behavior of the bio-inspired system resulting from inherent time delays and a time of arrival of the blast pressure wave. There is pointed out that the inherent state time delays change dynamical response of the multibody system. Proper time of deployment of the foam-based armor plate reduces relative compression of the thorax, which is to be protected by a bullet-proof waistcoat. 
Skočilas et al. propose a methodology that could be applied for investigation of central inertia moments and gravity center of large volume weight bodies, e.g. rail vehicles. Their methodology is applicable for various large dimensions of bodies and high weight machines. The results are useful in investigation of the dynamic stability of the movement and optimization of the construction of rail and road vehicles. The proposed methodology is based on the known fact that the inertia moment could be obtained from oscillation period of vibratory movement body, which is embedded on the rigid and elastic supports.

The problem of motion modeling and work cycle optimization of a manipulator with revolute joints has been considered by Posiadała et al. Equations of the manipulator's elements under any spatial work cycle conditions have been completed by using the classic vector mechanics and Lagrange equations of second kind. The objective function has been formulated using performance indexes and the design variables are rated velocity value and initial time value of work cycle in each considered actuator. The formulated optimization problem has been solved using constrained Multi-Objective Particle Swarm Optimization algorithm. A numerical computation has been completed using specially performed software.

Finite element modeling and prediction of the strength of the Shukhov's tower has been considered by Król and Olejarczyk. Four main frequencies and the vibration modes indicate the dominance of the local rigidity of the tower over the global stiffness. The usability criterion analysis of damage limits of the riveted joints in the Shukhov's tower points to exceed the limits in three of four criteria.

Wos and Dindorf present selected issues of position-force control of electro-hydraulic servo system using adaptive methods. This kind of measure extends the capabilities of control system which uses only position measurements. Indirect adaptive control allowed the authors for the monitoring and updating the dynamic phenomena occurring in the drive.
Coupled electromechanical interactions observed in a closed-loop control system actuated by a stepper motor are studied by Kępiński et al. Analyzed system consists of a PID controller used to maintain constant vertical inclination of a single DOF pendulum. This pendulum serves as a tensioner for a string being pulled by a rotary motion of the electric stepper motor. The second end of the string is being fed to the motor with a variable speed. The mathematical model governing dynamics of the mentioned system consists of a non-linear system of four ordinary differential equations. The rotor control using the micro-stepping approach has been used to model the real dynamics of the motor controller. Comparison of the proposed theoretical considerations and modelling of the system fits well with the experimental investigations.

Finally, it should be emphasized that the selected papers are mainly oriented toward modeling and identification of mechanical systems. They have been reviewed to satisfy the journal's standards. In addition, the guest-editors greatly appreciate a kind invitation of Professor Jian-Qiao Sun to publish the recommended papers within the thematic issue. Furthermore, a professional help of the IJDY's staff in the final production process is acknowledged.

Open Access This article is distributed under the terms of the Creative Commons Attribution 4.0 International License (http://creativecommons. org/licenses/by/4.0/), which permits unrestricted use, distribution, and reproduction in any medium, provided you give appropriate credit to the original author(s) and the source, provide a link to the Creative Commons license, and indicate if changes were made. 